Open Access

\title{
Neuropsychological assessment in adolescents with anorexia nervosa - exploring the relationship between self-report and performance-based testing
}

\author{
Kristin Stedal ${ }^{*}$ and Camilla Lindvall Dahlgren*
}

\begin{abstract}
Objective: Research investigating the relationship between neuropsychological test performances and selfreported cognitive functioning in patients with anorexia nervosa (AN) is limited, and existing experimental studies only demonstrate a low-to-moderate relationship between the performance based tests and everyday behaviour. The objective of the current study was to explore the association between neuropsychological test performance and self-reports of executive functioning in adolescents with AN.

Method: Twenty adolescent females diagnosed with AN, aged 13 to 18, completed neuropsychological test battery "the Ravello Profile" and the self-report version of the Behaviour Rating Inventory of Executive Function (BRIEF-SR). The BRIEF Parent Form (BRIEF-PF) was employed to provide additional information of the patients' executive functioning.
\end{abstract}

Results: Based on group level analyses, the results support the existing literature in failing to find consistent weaknesses in neuropsychological functioning in adolescents with AN. Further, with few exceptions, the Ravello Profile was insubstantially correlated with the majority of the BRIEF clinical scales, indicative of a lack of association between these two assessment methods.

Conclusion: The current study accentuates the need for concern regarding the generalizability of neuropsychological assessments in adolescent patients with AN.

Keywords: Anorexia nervosa, Eating disorders, Neuropsychological assessment, Ecological validity

\section{Background}

Clinicians working in the field of eating disorders will often describe patients with anorexia nervosa (AN) as being preoccupied with details, and displaying high levels of cognitive and behavioural rigidity. This pertains in particular to issues concerning food, weight and bodily appearance, but also to other aspects of everyday functioning like schoolwork and in relation to friends and family. In adults with AN, neuropsychological assessments and clinical observations have led researchers to hypothesise that there could be an underlying brain

\footnotetext{
*Correspondence: kristin.stedal@ulleval.no; camilla.lindvall@dahlgren.no Regional Department for Eating Disorders, Division of Mental Health and Addiction, Oslo University Hospital, Postboks 4956 Nydalen, 0424 Oslo,
} Norway

\section{Biomed Central

based abnormality causing this characteristic cognitive profile [1-5], and that such abnormalities could represent an AN endophenotype rather than temporary, state dependent cognitive functioning [6-12]. In adolescents with AN, however, most research fails to find consistent neuropsychological weaknesses [13-16] and two recent reviews on set-shifting and central coherence support the notions that such difficulties are less pronounced in this patient group [17, 18].

Several hypotheses have been put forward to explain why the neurocognitive profile of young AN patients differs from that of adults. According to Bühren et al. $[13,19]$, set shifting in children and adolescents is a skill that has yet to evolve, and the brain maturation, especially the cortico-striatal networks which modulate 
set-shifting, are not fully developed until early thirties, and thus, cannot be labelled defective. Previous studies have also suggested weaknesses in neuropsychological functioning as being a consequence of the illness, and representing a scar effect with its severity depending on the duration of illness [19-21]. In line with this hypothesis, it is possible that, although many young patients with AN do not exhibit neuropsychological difficulties, such will emerge as the illness progress, and hence, be detectable as the course of the illness extends. It could also be argued that the instruments used to assess neuropsychological functioning fail to capture cognitive challenges as they appear in contextually meaningful settings. Clinically, many young individuals with AN undoubtedly struggle with being flexible and being able to see "the bigger picture", especially in terms of eating disorder (ED) specific symptoms such as weight, shape, food and purging behaviours. However, as these weaknesses do not always appear to manifest in a controlled test-setting, this might indicate low sensitivity of assessment measures, that is, that they are not sensitive enough to pick up on modest neuropsychological weaknesses, or perhaps even more realistic, that rigidity and detail focus related to everyday life activities are nonassessable using neuropsychological tests, i.e., the tests lack ecological validity.

Neuropsychological assessments were historically employed as a way of localising and detecting neuropathology. Based on an individual's cognitive profile of strengths and weaknesses, inferences would be drawn regarding brain dysfunctions and the probable aetiology [22]. It is frequently assumed that results from neuropsychological performance tests can predict every day functioning [23], and it is commonly expected that poor performance on a neuropsychological test will be of relevance for clinical purposes and in terms of the patient's day-to-day functioning. Surprisingly, there has been very little research investigating the accuracy of this assumption, and existing experimental studies recurrently demonstrate only a weak-to-moderate relationship between the performance on neuropsychological tests and everyday behaviour [24-27]. Further, performance-based tests of executive functioning have been suggested to be more sensitive to deficits in adults than in children, as they were originally developed for use with adult populations [28].

In 2004 a neuropsychological test battery named "the Ravello Profile" was developed as an assessment tool specifically aimed at investigating cognitive functioning, and in particular, various aspects of executive functioning in patients with AN [29]. Several studies employing this test battery in AN populations have been published $[16,30-32]$, but the results so far have only demonstrated small to insignificant impairments in adolescents with AN
[16, 30-32]. This is in opposition to the previous literature on cognitive functioning in adults with AN, but perhaps even more pertinent, in complete contrast to how clinicians, parents and teachers describe these patients in terms of rigidity and preoccupation with detail, order and symmetry. As quantifying everyday cognitive functioning is a complex task, and as any form of assessment is associated with a certain degree of error [33], one might be inclined to ask oneself how to most accurately assess neuropsychological function in adolescents with AN. Recently, novel measures assessing executive functions in contextually relevant settings have emerged with the aim of complementing test-based results and increase ecological validity. The Behavior Rating Inventory of Executive Function (BRIEF) [34] was developed as a tool for assessing executive functioning in children and adolescents in an ecologically valid manner. It yields evidence of executive function behaviours in school and home environments, and can serve as an important adjunct to clinical evaluation and neuropsychological assessments of executive dysfunction [35]. The BRIEF has been extensively employed in assessing executive functions in attention deficit hyperactivity disorder (ADHD) [36-38] and in traumatic brain injury research $[39,40]$, but its use in research on eating disorders has so far been limited [41, 42].

The aim of the current study was to explore the relationship between performance based- and self-reported neuropsychological functioning in adolescents with AN. The means through which this was achieved, was by comparing tests results derived from the Ravello Profile subtests, and self- and parent reports of executive functioning assessed using the BRIEF-SR [43] and BRIEF-PF $[34,44]$.

\section{Method \\ Participants}

Data were collected as part of a feasibility trial of cognitive remediation therapy (CRT) for adolescents with AN $[16,41]$. The sample consisted of 20 female in- and outpatients currently in treatment for AN. Patients were between 13 and 18 years of age (Mean =15.9, $S D=1.6$ ) and ethnic Norwegian. Ten of the patients were recruited from a paediatric outpatient service, and the remaining ten from a regional inpatient service. At time of assessment, inpatients were hospitalized and receiving high-intensity physical and psychological treatment. The outpatients were engaged in less intense treatment, primarily oriented towards physical aspects of the illness. The Regional Committee for Medical Research Ethics (REK) granted ethical approval to conduct the study. All patients were fully informed about the study, and gave written informed consent. Parental consent was obtained for patients below the age of 16 . 
There were no significant differences between in- and outpatients on any of the baseline assessment variables. Patients' weight and height was measured in conjunction with assessments, with results revealing a mean BMI percentile just above $10 \%($ Mean $=10.2, S D=17.2)$. The Eating Disorder Examination Questionnaire version 6.0 (EDE-Q 6.0) [45] was used to assess eating disorder psychopathology, with results yielding a mean global EDE$\mathrm{Q}$ score of 3.4 $(S D=1.4)$. Based on the results from the EDE-Q assessing binge eating and compensatory behaviours, 18 patients were classified as having a restricting subtype of AN (AN-R), and the two remaining patients fitted the description for a binge-purge subtype (AN-BP). Verbal intelligence was assessed using the Vocabulary subtest from the Wechsler Adult Intelligence Scale - Third Edition (WAIS-III) [46], and the Wechsler Intelligence Scale for Children - Third Edition (WISC-III) [47]. Performance-IQ was assessed using the Matrix Reasoning subtest from the WAISIII, and the Wechsler Abbreviated Scale of Intelligence (WASI) [48]. All patients scored within the normal range on the measures assessing intelligence.

\section{Assessment \\ Self-report measures BRIEF-SR}

The self-report version of the Behavior Rating Inventory of Executive Function (BRIEF-SR) [43] yields information about young people's views of their own executive functions (EF) in every day life settings, and is designed for children and adolescents between the ages of 11 and 18. The 80-item questionnaire is scored on a 3-point Likert scale: "never", "sometimes", and "always," and provides scores on eight clinical scales describing various aspects of EF. Four subscales, Inhibit, Shift, Emotional Control and Monitor, are combined to produce a broader index called the Behavior Regulation Index (BRI). The four remaining subscales, Working memory, Plan/Organize, Organization of materials and Task completion, comprise the Metacognitive Index (MI). The Global Executive Composite (GEC) is the overall score, which is a composite of the two index scores. Scaled BRIEF-SR scores are transformed to age and gender corrected t-scores $(M=50$, $\mathrm{SD}=10$ ), for which higher scores indicate higher degrees of executive dysfunction. T-scores at or above 65 (i.e. 1.5 SD above the normative mean) are considered clinically significant in terms of executive dysfunction.

\section{BRIEF-PF}

The BRIEF Parent Form (BRIEF-PF) [34, 44] is to be completed by the caregiver, and is an 86 -item questionnaire designed to provide detailed knowledge of the child's behaviour. It overlaps with the BRIEF-SR on the following subscales: Inhibit, Shift, Emotional control,
Working memory, Plan/Organize, Organization of materials and Monitor. Adding a ninth subscale labelled "Initiation", these clinical subscales together produce the same two indexes as the BRIEF-SR: the BRI and the MI, as well as a composite score (GEC). Scoring procedures are identical for the BRIEF-PF and BRIEF-SR. The BRIEF-SR and BRIEF-PF clinical scales are described in more detail in Table 1.

Both BRIEF-SR and BRIEF-PF are standardized assessment instruments with well-established psychometric properties [43]. For the BRIEF-SR, high internal consistency has been shown for the 80-item GEC (.96), and test-retest reliability is supported by correlations among clinical scales ranging from .59 to .85 . Higher correlations were observed for the index scores BRI and MI (.84 and .87 respectively), with the highest correlation (.89) emerging for the GEC [49]. Convergent evidence of validity is further supported by moderate correlations (for GEC, $r=.56$ ) between BRIEF-SR and BRIEF-PF. For the BRIEF-PF, high internal consistency has been reported $(.80-.98)$, and test-retest correlation ranging from .81 (clinical scales) to .88 (GEC) with the highest correlation emerging for the metacognitive index (MI) (.88) [34]. Further, preliminary analyses have supported its reliability and validity in assessing EF in adults with AN [50]

\section{Neuropsychological Assessment}

The neuropsychological test battery "the Ravello Profile" $[29,51]$ (Table 2) was developed specifically to assess individuals with $\mathrm{AN}$, and is suitable for individuals between the ages of $8-89$. The test battery includes a variation of subtest from the Delis Kaplan Executive Functioning System (D-KEFS) [52], the Rey Complex Figure Test (RCFT) [53, 54], and the Brixton Test [55]. For more detailed description and information regarding the Ravello Profile, see Rose et al. [29, 51].

\section{Procedure}

Patients and parents completed the BRIEF-SR and BRIEFPF questionnaires in conjunction with the patients' neuropsychological assessment (i.e. the Ravello Profile), which were administered by two trained investigators. A total of 14 mothers and 6 fathers completed the BRIEF-PF.

\section{Statistical Analyses}

All statistical analyses were carried out using PASW $^{\odot}$ Statistics 18 for Windows XP/Vista ${ }^{\circ}$. For comparative purposes, the neuropsychological raw scores were converted to $z$-scores using means and SDs using the tests' published age-based norms. Z-scores have a mean of zero and a standard deviation of 1 . Exceptions were the Brixton Spatial Anticipation Test and the Central Coherence measure for which norms for children and 
Table 1 BRIEF-SR \& BRIEF-PF clinical scales

\begin{tabular}{|c|c|}
\hline Clinical scale & Description and intrpretations of scale \\
\hline Inhibit & $\begin{array}{l}\text { The ability to inhibit, resist or not act on an impulse. Also refers to the ability to stop one's own behavior } \\
\text { at an appopriate time. }\end{array}$ \\
\hline Shift & $\begin{array}{l}\text { The content-dependent ability to move freely from one activity, situation or aspect of a problem to another. } \\
\text { Includes making swift transitions, problem-solve flexibly and switch or alternate attention. }\end{array}$ \\
\hline Behavioral shift ${ }^{1}$ & To be able to flexibly alter your behavior depending on environment or schedule \\
\hline Cognitive shift ${ }^{1}$ & To flexibly solve problems \\
\hline Emotional control ${ }^{1}$ & The ability to adjust emotional responses in an appropriate and constructive way \\
\hline Monitor & $\begin{array}{l}\text { The ability of the child to assess its own performance to attain knowledge of progress in terms of personal goals } \\
\text { and achievements. Also a personal monitoring functioning to ensure the effect on one's own behaviors on others }\end{array}$ \\
\hline Working memory & The ability to hold information in mind with the purpose of completing a task or activity \\
\hline Plan/Organize & $\begin{array}{l}\text { The ability to tackel demands, both current and future-oriented. To be able to anticipate future demands, to set } \\
\text { goals and to time-efficiently develop strategies for goal achievement }\end{array}$ \\
\hline Organization of materials & The orderliness of work, play and storage spaces (bedrooms, lockers, desks etc.) \\
\hline Task completion ${ }^{1}$ & The ability to finish or complete task appropriately and within a given timeframe \\
\hline Initiate $^{2}$ & The ability of initiation an assignment or activity. To be able to independently generate ideas. \\
\hline
\end{tabular}

${ }^{1}=$ Only in BRIEF-SR

${ }^{2}=$ Only in BRIEF-PF

adolescents are not available. Consequently, control group means from a recently published study employing these measures in a child and adolescent sample were used $(\mathrm{N}=66)$ [31]. The relationship between neuropsychological performance (as measured by the tests in the
Ravello Profile) and self-reports of executive functioning (as measured by the BRIEF-SR and BRIEF-PF scales) was investigated using the Spearman rank order correlations rho. Rho $(\rho)$ is defined as small (.10 to .29), medium (.30 to .49 ) or large (.50 to 1.0$)$. Due to the exceptionally large

Table 2 The Ravello Profile

\begin{tabular}{lll}
\hline Domain & Task & Assesment material \\
\hline IQ & & WAIS-III / WASI \\
Verformance IQ & Matrix Reasoning & WAIS-III / WASI-III \\
Executive Functioning & Vocabolary & D-KEFS \\
Cognitive Inhibition & Colour Word Interference Condition 3 & D-KEFS \\
Cognitive Inhibition \& Flexibility & Colour Word Interference Condition 4 & D-KEFS \\
Verbal Fluency & Verbal Fluency Condition 1 & D-KEFS \\
Verbal Fluency & Verbal Fluency Condition 2 & D-KEFS \\
Switching & Verbal Fluency Condition 3 & D-KEFS \\
Switching & Trial Making Test Condition 4 & D-KEFS \\
Planning \& Inhibition & Tower of London & Hayling \& Brixton \\
Flexibility \& Spatial Working & Brixton Spatial Anticipation Test & RCFT \\
Memory & & RCFT \\
Visio-Spatial Memory & Immediate Recall & RCFT \\
Visual Spatial Processing & Delayed Recall & RCFT \\
& RCFT Style Index & RCFT \\
\hline
\end{tabular}


Table 3 The Ravello Profile, BRIEF-SR and BRIEF PF z-scores ( $\mathrm{N}=20)$

\begin{tabular}{|c|c|c|c|c|c|c|c|c|c|c|c|c|}
\hline \multicolumn{13}{|c|}{ Ravello Profile mean z-scores (SD) - patients } \\
\hline RCFT & RCFT & RCFT & Verbfl3 & TMT4 & Stroop3 & Stroop4 & Tower & Brixton & RCFT & RCFT & RCFT & \\
\hline Immediate & Delayed & Recogn. & & & & & & & Style & Order & $\mathrm{CCl}$ & \\
\hline$-.61(1.23)$ & $-.69(1.38)$ & $-.37(1.35)$ & $.68(.90)$ & $-.65(.76)$ & $-.32(1.15)$ & $.00(86)$ & $.42(.71)$ & $.12(1.28)$ & $.55(.7)$ & $.48(1.02)$ & $.64(.79)$ & \\
\hline \multicolumn{13}{|c|}{ BRIEF-SR mean z-scores (SD) - patients } \\
\hline & & Behavioral & Cognitive & Emotional & Working & Plan/ & Org. of & Task & & & & \\
\hline Inhibit & Shift & shift & shift & control & Memory & Organise & material & completion & Monitor & BRI & $\mathrm{Ml}$ & GEC \\
\hline$-.06(1.34)$ & $1.44(1.39)$ & $1.42(1.63)$ & $.93(1.28)$ & $1.23(1.22)$ & $.19(1.37)$ & $.03(1.16)$ & $-.39(1.07)$ & $.57(1.60)$ & $-.16(1.02)$ & $.83(1.32)$ & $.08(1.46)$ & $.54(1.42)$ \\
\hline \multicolumn{13}{|c|}{ BRIEF-PF mean z scores (SD) - parents } \\
\hline & & & Emotional & Working & Plan/ & Organization of & & & & & & \\
\hline Inhibit & Shift & Initiate & control & memory & organise & materials & Monitor & BRI & Ml & & GEC & \\
\hline $.12(1.12)$ & $1.20(1.08)$ & $.21(1.01)$ & $1.52(1.12)$ & $.46(1.28)$ & $.33(1.06)$ & $-.77(.80)$ & $.18(.79)$ & $1.11(1.03)$ & $.18(.93)$ & & $.55(.89)$ & \\
\hline
\end{tabular}

$S D=$ Standard Deviation, BRIEF SR = Behavioral Rating Inventory of Executive Funtion -Self Report, BRIEF-PF = Behavioral Rating Inventory of Executive Funtion - Parent Form, $R C F T=$ Rey Complex Figure Test, Verbfl = Verbal Fluency, $T M T=$ Trial Making Test, Style = Style index, Order = Order of construction index, $C C l=$ Central coherence index, BRI = Behavioral Regulation Index, $M I=$ Metacognitive Index, GEC = Global Executive Composite 
Table 4 Spearman rank order correlations $(\rho)$ between the Ravello Profile tests and the BRIEF-SR scales $(N=20)$

\begin{tabular}{|c|c|c|c|c|c|c|c|c|c|c|c|c|c|c|}
\hline \multicolumn{15}{|c|}{ BRIEF-SR } \\
\hline & & Inhibit & Shift & $\begin{array}{l}\text { Emotional } \\
\text { control }\end{array}$ & Monitor & $\begin{array}{l}\text { Working } \\
\text { memory }\end{array}$ & $\begin{array}{l}\text { Plan/ } \\
\text { Organise }\end{array}$ & $\begin{array}{l}\text { Organisation } \\
\text { of materials }\end{array}$ & $\begin{array}{l}\text { Task } \\
\text { completion }\end{array}$ & BRI & $\mathrm{Ml}$ & GEC & $\begin{array}{l}\text { Behavioral } \\
\text { Shift }\end{array}$ & $\begin{array}{l}\text { Cognitive } \\
\text { shift }\end{array}$ \\
\hline \multirow{16}{*}{$\begin{array}{l}\text { The Ravello } \\
\text { Profile }\end{array}$} & RCFT Immediate & .19 & -.01 & -.07 & .08 & .06 & .36 & .14 & .30 & .07 & .18 & .25 & .03 & -.10 \\
\hline & RCFT Delayed & .17 & .03 & .02 & .08 & .01 & .40 & .09 & .29 & .10 & .15 & .23 & .10 & -.05 \\
\hline & RCFT Recognion & .07 & .09 & .06 & .03 & -.09 & .10 & -.12 & -.05 & .10 & -.06 & -.02 & .07 & .11 \\
\hline & RCFT Style & -.07 & .02 & -.17 & -.12 & -.06 & .24 & -.10 & .25 & -.09 & .05 & .07 & .03 & .01 \\
\hline & RCFT Order & .10 & .04 & .05 & .11 & .36 & .49 & .23 & .50 & .06 & .46 & .34 & -.04 & .16 \\
\hline & RCFT CCl & .22 & .12 & .02 & .14 & .25 & .39 & .31 & .36 & .12 & .35 & .35 & .02 & .22 \\
\hline & Verbal Fluency 1 & .26 & .14 & .10 & -.07 & .04 & -.02 & -.01 & -.35 & .14 & -.20 & .08 & .12 & .08 \\
\hline & Verbal Fluency 2 & .10 & .04 & .23 & .03 & -.20 & -.09 & -.08 & -.26 & .13 & -.20 & -.04 & .22 & -.16 \\
\hline & Verbal Fluency 3 & -.00 & -.16 & -.11 & -.07 & .05 & .09 & -.23 & .05 & -.10 & -.18 & -.02 & -.14 & -.23 \\
\hline & Trail Making Test 4 & .24 & -.19 & .11 & -.00 & .00 & -.04 & -.06 & -.41 & .15 & -.18 & .06 & -.15 & .01 \\
\hline & Stroop 3 & .49 & .16 & .37 & .36 & .11 & .10 & .16 & -.16 & .44 & -.00 & .20 & .04 & .08 \\
\hline & Stroop 4 & .37 & .31 & .18 & .08 & -.06 & .07 & .06 & -.16 & .36 & -.25 & .19 & .17 & .18 \\
\hline & Tower & -.27 & -.48 & -.45 & -.27 & -.26 & -.53 & -.16 & -.19 & -.45 & -.22 & -.48 & -.50 & -.39 \\
\hline & Brixton & -.16 & -.22 & -.29 & -.10 & -.09 & .05 & -.30 & -.07 & -.23 & -.20 & -.06 & -.07 & -.36 \\
\hline & WAIS/WASI Matrix & .44 & -.17 & .09 & .21 & .09 & -.04 & -.03 & -.26 & .19 & -.05 & .07 & -.16 & -.19 \\
\hline & WAIS/WISC Vocabulary & -.10 & -.03 & -.19 & -.33 & -.15 & -.02 & -.20 & -.20 & -.11 & -.33 & -.10 & -.21 & .07 \\
\hline
\end{tabular}

All correlations are based on $z$-scores. Spearman's rank order correlations ( $\rho)$ are defined as small (.10 to .29), medium (.30 to .49$)$ or large (.50 to 1.0$)$. Large correlations (.50 $\geq)$ are marked in bold.

$B R I E F-S R=$ Behavior Rating Inventory of Executive Function - Self-Report, $R C F T=$ Rey Complex Figure Test, Immediate = Immediate recall, Delayed = Delayed recall, Style = Style index, Order = Order of construction index, $C C I=$ Central coherence index, WAIS = Wechsler Adult Intelligence Scale, WASI = Wechsler Abbreviated Scale of Intelligence, WISC = Wechsler Intelligence Scale for Children, $B R I=$ Behavior Regulation Index $M I=$ Metacognitive Index,$G C E=$ Global Executive Composite 
Table 5 Spearman rank order correlations $(\rho)$ between the Ravello Profile tests and the BRIEF-PF scales $(N=20)$

\begin{tabular}{|c|c|c|c|c|c|c|c|c|c|c|c|c|}
\hline \multicolumn{13}{|c|}{ BRIEF-PF } \\
\hline & & Inhibit & Shift & $\begin{array}{l}\text { Emotional } \\
\text { control }\end{array}$ & Initiate & $\begin{array}{l}\text { Working } \\
\text { memory }\end{array}$ & $\begin{array}{l}\text { Plan/ } \\
\text { organise }\end{array}$ & $\begin{array}{l}\text { Organisation } \\
\text { of materials }\end{array}$ & Monitor & $\mathrm{BRI}$ & $\mathrm{Ml}$ & GEC \\
\hline \multirow{16}{*}{$\begin{array}{l}\text { The Ravello } \\
\text { Profile }\end{array}$} & RCFT Immediate & -.10 & .25 & .08 & .08 & .09 & .17 & -.07 & .15 & .11 & .11 & .13 \\
\hline & RCFT Delayed & -.08 & .19 & .01 & .17 & .00 & .12 & .03 & .11 & .03 & .12 & .10 \\
\hline & RCFT Recognion & .13 & .10 & .32 & .11 & -.18 & -.12 & .25 & -.02 & .17 & -.02 & .02 \\
\hline & RCFT Style & -.25 & .14 & .01 & .27 & .10 & .19 & -.02 & -.14 & .03 & .18 & .15 \\
\hline & RCFT Order & -.25 & .16 & -.17 & .20 & .21 & .29 & .27 & .02 & -.13 & .28 & .14 \\
\hline & RCFT CCl & -.27 & .04 & .20 & .19 & .20 & .21 & .32 & -.01 & -.12 & .26 & .13 \\
\hline & Verbal Fluency 1 & .37 & -.10 & .06 & -.20 & -.15 & -.03 & -.13 & .16 & .15 & -.07 & .00 \\
\hline & Verbal Fluency 2 & .31 & .12 & .23 & .04 & -.08 & -.07 & -.31 & .36 & .20 & -.01 & .07 \\
\hline & Verbal Fluency 3 & .30 & .06 & .10 & -.26 & -.30 & -.13 & -.44 & -.04 & .28 & -.27 & -.08 \\
\hline & Trail Making Test 4 & -.02 & -.37 & -.22 & -.45 & -.63 & -.43 & -.19 & -.14 & -.24 & -.51 & -.47 \\
\hline & Stroop 3 & -.17 & -.23 & -.11 & -.26 & -.48 & -.40 & -.25 & -.14 & -.23 & -.42 & -.42 \\
\hline & Stroop 4 & .04 & -.28 & -.08 & -.26 & -.39 & -.15 & -.32 & -.24 & -.05 & -.35 & -.27 \\
\hline & Tower & -.57 & -.08 & -.20 & -.09 & .04 & -.06 & .09 & -.36 & -.27 & -.09 & -.14 \\
\hline & Brixton & .30 & .21 & .19 & -.12 & .04 & .19 & -.33 & -.04 & .40 & -.01 & .17 \\
\hline & WAIS/WASI Matrix & .21 & .25 & .35 & -.08 & -.22 & -.26 & -.03 & .02 & .33 & -.21 & .01 \\
\hline & WAIS/WISC Vocabulary & -.22 & -.38 & -.21 & -.45 & -.56 & -.19 & -.19 & -.35 & -.31 & -.45 & -.44 \\
\hline
\end{tabular}

All correlations are based on $z$-scores. Spearman's rank order correlations ( $\rho$ ) are defined as small (.10 to .29), medium (.30 to .49) or large (.50 to 1.0). Large correlations (.50 $\geq$ ) are marked in bold.

$B R I E F-P F=$ Behavior Rating Inventory of Executive Function - Parent Form, RCFT = Rey Complex Figure Test, Immediate = Immediate recall, Delayed = Delayed recall, Style = Style index, Order = Order of construction index, $\mathrm{CCI}=$ Central coherence index, WAIS = Wechsler Adult Intelligence Scale; WASI = Wechsler Abbreviated Scale of Intelligence, WISC = Wechsler Intelligence Scale for Children, BRI = Behavior Regulation Index, $M I=$ Metacognitive Index; GCE = Global Executive Composite 
number of correlations $(\mathrm{N}=384)$, we chose to focus on the magnitude of correlations rather than their statistical significance. Only large correlations $(.50 \geq)$ were considered being of interest.

\section{Results \\ Neuropsychological functioning, BREIF-SR \& BRIEF-PF scores}

Table 3 present $z$-scores on the Ravello Profile subtests, and on the BRIEF-SR \& BRIEF-PF subscales. As evident from the table, all neuropsychological test scores fell within the normal range. This was also true for both BRIEF-SR and BRIEF-PF scores (correspondent $t$-scores have been presented elsewhere [41]).

\section{Correlational analysis}

Negative medium correlations were observed for the Tower task and five BRIEF-SR subscales. The results also revealed a large positive correlation between the RCFT, OCI and the BRIEF-SR scale Task completion, and medium correlations between scores on WAIS/WISC Vocabulary and two BRIEF-SR subscales. As for correlations between parents' reports of their children's executive functions (BRIEF-PF) and the children's neuropsychological test performance, the correlational analysis revealed large negative correlations between the TMT and two BRIEF-PF subscales, a large negative correlation between the Tower task and the BRIEF-PF inhibit subscale, as well as medium to large negative correlations between WAIS/WISC Vocabulary and a number of subscale and composite BRIEFPF scales. Details are presented in Table 4 and 5.

\section{Discussion}

To the authors knowledge, this is the first study investigating the relationship between a neuropsychological assessment method specifically aimed at assessing patients with AN (i.e. the Ravello Profile) and self-reports of executive functioning. The study highlights the fact that the conceptualizations behind these test were to aid in the diagnosis of neuropathology - not as means of predictions about the functioning of psychiatric populations in real-life or treatment settings [56]. The results are in line with previous studies demonstrating only a low-to-moderate relationship between reports of everyday skills and scores on neuropsychological tests [57-59], and support the existing literature in failing to find consistent weaknesses in neuropsychological functioning in adolescents with AN. The results further accentuate the need for concern regarding the generalizability of neuropsychological assessments in adolescent patients with AN.

On an overall level, there was a lack of correlations between the tests in the Ravello Profile and the BRIEF-SR and BRIEF-PF clinical subscales. This lack of correlations could be attributed to a variety of interpretations. Firstly, it is possible that the two assessment methods simply measure different types of executive functions. Secondly, there is a risk that BRIEF reports might be biased due to the nature of patient care for half of the patients (i.e. inpatients) making it difficult for patients and parents to assess "normal" day-to-day behaviour. Further, as the two assessment methods vary greatly, it is not unlikely that factors such as personality, environmental influence and personal efforts had an uneven impact on performance based and self-reported scores.

However, there were some exceptions. The results demonstrate an association between the WAIS/WISC vocabulary subtest and several of the BRIEF-SR and BRIEF-PF subscales. These findings are comparable to those of Vriezen and Pigott [28] who investigated the relationship between the parental report of the BRIEF and performance based measures of executive function in children with moderate to severe traumatic brain injury. Results from this study revealed that the BRIEF did not correlate with any of the traditional performance-based tests of executive function (the Trail making test $\mathrm{B}$, the Verbal fluency test and the Wisconsin card sorting test), but that verbal intelligence as measured by the WISC-III correlated with metacognitive aspects of executive functioning such as initiation, organisation and monitoring of activities [28]. The Vocabulary subtest of the WAIS/ WISC has previously been shown to be closely related to overall verbal intelligence $[60,61]$. The current study yields support to the strong association between verbal abilities and the capability to monitor and assess performance, and to acquire knowledge in terms of personal goals and achievements.

Further, it is of interest to note that the Tower test was correlated with a number of the BRIEF-SR scales such as Shift, Behavioral shift, Emotional control and Plan/Organize. This is in line with previous studies suggesting that the Tower test is a complex task requiring multiple aspects of executive functioning, including planning [62, 63], inhibition [64] and working memory $[65,66]$. In the current study, Tower test performance was related to the ability to flexibly shift and alternate attention, to track demands - both current and future oriented - as well as to keep emotional control during a rather stressful and demanding task. The large correlation between the Tower test and these BRIEF-SR subscales also indicates that the Tower test might be a neuropsychological performance test which is highly applicable for assessing executive functioning in patients with AN. The Tower test measures several aspects of functioning, and appears to be a more ecologically valid instrument compared to some of the other tasks included in the Ravello Profile. This is in line with previous studies demonstrating that tests, which assess 
several executive domains simultaneously, are more similar to life-like challenges [67].

The majority of tests used in studies assessing neuropsychological functioning in patients with AN are instruments with a long history within the field of neuropsychology. The choice of specific tests is often based on its professed ability to assess specific cognitive domains, for example executive functioning. However, due to the fact that the majority of such tests have an inherent complexity, few of them actually measure merely a single function or ability. Also, poor performance on individual tasks could be the result for a variety of sub-optimal functioning skills [22], rather than a specific weakness. The results from studies investigating neurocognitive functioning in children and adolescents with AN have been highly inconsistent, and it is probable that the variability in results could be due to performance-based tests of executive functioning being more sensitive to deficits in adults than in children [28]. Further, albeit being sensitive in discriminating participants with a brain injury from controls [22], neuropsychological tests might not be particularly effective when it comes to predicting every day difficulties. Thus, test results within the normal range do not necessarily imply evidence of absence of abnormal brain functioning [68]. In addition, traditional neuropsychological tests do not take into account factors like; personality, the support from family and surroundings, as well as performance motivation, which can make them less able to predict real-life performance [68].

Because of the complex nature of executive functioning there are many difficulties associated with the assessment of these skills - perhaps in particular when attempting to say something about its relevance to cognitions and behaviours relevant to the everyday life of patients $[23,69,70]$. A neuropsychological test performance falling within the norm could mask a greater effort required by a person suffering from sub-optimal brain functioning. Studies have demonstrated that children can perform well on standardized neuropsychological tests whilst still having difficulties with everyday activities demanding strong executive functioning capacities [71]. Neuropsychological assessments are usually conducted free of distractions, in a quiet room with a test administrator who will carefully explain the rules, set goals and initiate and stop behaviours where appropriately [72]. This means that the testing environment itself can ameliorate difficulties with executive functions like starting and stopping behaviours, completing tasks and staying focused on the test being presented. Thus, a possible interpretation of the findings on the Ravello Profile in this study is that the structured setting of the neuropsychological assessment situation aids the patients performance, whilst the BRIEF provides an assessment of executive functioning which more accurately represents true day-to-day day functioning.

To the authors knowledge this is the first study to explore the relationship between Ravello Profile subtests and self-reports of executive functioning in adolescents with AN. However, the findings should be treated by caution as they are limited by a small sample size and the potential of truncated distribution of scores. Future studies should increase the number of participants to strengthen the validity and generalizability of results. The use of a self-report measure is a further limitation when interpreting the findings from this study. Illness and self-awareness is closely related to executive functions, and there is a tendency for patients with executive functioning difficulties to underestimate their own problems [73], thus the self-report version of the BRIEF (BRIEF-SR) might alone not be adequate for assessing impaired executive functioning in young patients with AN. Rather, by supplementing the BRIEF-SR with the report of relatives and/or teachers, the participants difficulties might be more accurately determined. Discrepancies between BRIEF-SR and BRIEF-PF ratings in the current population have been previously described by Dahlgren et al. [41] supporting the need for informant ratings of executive functioning in adolescents with AN. The Ravello Profile, the BRIEF-SR and the BRIEF-PF scores were all below the clinical cut-off level when analysed by group mean scores. This highlights the notion that, although these patients are commonly described as displaying executive dysfunctional behaviours and rigid cognitions, the scores on both the performance based tasks and behaviour rating inventories are within the normal range. As the BRIEF was developed specifically to yield ecologically valid measures of executive functions, one would expect it to be fairly sensitive to such difficulties in day-to-day functioning [44]. However, it has been demonstrated that there is great variability in scores on neuropsychological tests of executive functioning in patients with $\mathrm{AN}$, and that individuals who struggle with these tasks can be masked when performing whole group analyses [30]. Future studies of executive functioning in adolescent patients with AN should aim at emphasizing assessments more closely related to real life, and to place grater focus on observations of behaviours of structured tasks performed in real world settings. Naturalistic assessment procedures, like the Multiple Errands Test [74], is an example of an approach where the aim is to obtain a greater understanding of the participants' real world functioning. Some preliminary work on assessments of executive functions using virtual reality has also been proposed [75, 76], and could be helpful for improving ecological validity in assessing executive functions in this patient group. Combining performancebased tests with information from behavioural observations, interviews, informant ratings and self-report 
measures could enhance its relevance to everyday life. When employing a combination of neuropsychological performance tests and self-reports, difficulties experienced by patients can be further delineated, and psychological interventions can be better targeted. By taking a broad minded approach to neurocognitive assessments of young patients with $\mathrm{AN}$, the possibility of better targeted interventions aimed at ameliorating cognitive challenges in the everyday life of the patients can be facilitated. Further, there is a lack of assessment tools targeting the evaluation of executive dysfunction in relation to ED specific cognitions and behaviours such as body shape and weight. At this point, patients with AN are often described as rigid and preoccupied with details, but researchers have yet to clarify whether such cognitive and behavioural characteristics pertains to the eating disorders per se, or if such categorizations reflects alterations in global executive function.

Finally, it is worth pointing out that the study holds some limitations with regards to the use of neuropsychological tests to reflect every day function. As mentioned previously, the tests used are based on a deficit model aiming to identify and quantify cognitive deficits, and might not be entirely suitable for the study of functional problems of people with anorexia nervosa scoring within the normal range. We can therefore not draw definite conclusions, and future studies are warranted to address this issue.

\section{Conclusions}

The neuropsychological test battery, the Ravello Profile, was insubstantially correlated with the majority of the BRIEF clinical subscales, accentuating the need for concern regarding the generalizability of neuropsychological assessments in adolescent patients with AN. Future studies should aim to increase the sample size in order to be able to generalize findings, control for multiple comparisons and, potentially, look at executive functioning directly related to eating disorder psychopathology.

\section{Competing interests}

The authors declare that they have no competing interests.

\section{Authors' contributions}

KS participated in the design of the study and drafted the manuscript. CLD performed the statistical analysis and participated in drafting the manuscript. All authors read and approved the final manuscript.

\section{Acknowledgements}

The work on this paper has been supported by the Regional Department for Eating Disorders, Division of Mental Health and Addiction, Oslo University Hospital, Ullevål. The authors would also like to thank the participants for their valuable contribution, and the reviewers for constructive comments.

Received: 12 January 2015 Accepted: 30 June 2015

Published online: 13 August 2015

\section{References}

1. Phillipou A, Rossell SL, Castle DJ. The neurobiology of anorexia nervosa: A systematic review. Aust NZ J Psychiat. 2014;48:128-52.

2. Sato Y, Saito N, Utsumi A, Aizawa E, Shoji T, Izumiyama M, et al. Neural Basis of Impaired Cognitive Flexibility in Patients with Anorexia Nervosa. PLoS ONE. 2013;8:5.

3. Nagamitsu S, Araki Y, loji T, Yamashita F, Ozono S, Kouno M, et al. Prefrontal brain function in children with anorexia nervosa: a near-infrared spectroscopy study. Brain Dev. 2011;33:35-44.

4. Hatch A, Madden S, Kohn MR, Clarke S, Touyz S, Gordon E, et al. Emotion brain alterations in anorexia nervosa: a candidate biological marker and implications for treatment. J Psychiatry Neurosci. 2010;35:267-74.

5. Zastrow A, Kaiser S, Stippich C, Walther S, Herzog W, Tchanturia K, et al. Neural correlates of impaired cognitive-behavioral flexibility in anorexia nervosa. Am J Psychiat. 2009;166:608-16.

6. Zucker NL, Losh M, Bulik CM, LaBar KS, Piven J, Pelphrey KA. Anorexia nervosa and autism spectrum disorders: guided investigation of social cognitive endophenotypes. Psychol Bull. 2007;133:976-1006.

7. Idini E, Marquez-Medina D, Pifarre J, Buj-Alvarez I, Castan-Campanera E. Are the neuropsychological alterations in eating disorders endophenotypes of the disease? Review and state of the art. Son las alteraciones neuropsicologicas de los trastornos de la conducta alimentaria endofenotipos de la enfermedad? Revision y estado actual del tema. Rev Neurol. 2012;55:729-36.

8. Galimberti E, Sani V, Scavelli V, Fadda E, Fanini F, Bellodi L. Executive dysfunctions in anorexia nervosa patients, obsessivecompulsive patients and their unaffected relatives: Searching for a new intermediate phenotype. European PsychiatryConference: 19th European Congress of Psychiatry, EPA 2011. Vienna Austria: Conference Publication; 2011.

9. Galimberti E, Fadda E, Cavallini MC, Martoni RM, Erzegovesi S, Bellodi L. Executive functioning in anorexia nervosa patients and their unaffected relatives. Psychiatry Res. 2013;208:238-44.

10. Holliday J, Tchanturia K, Landau S, Collier D, Treasure J. Is impaired setshifting an endophenotype of anorexia nervosa? Am J Psychiat. 2005;162:2269-75.

11. Tenconi E, Santonastaso P, Degortes D, Bosello R, Titton F, Mapelli D, et al. Set-shifting abilities, central coherence, and handedness in anorexia nervosa patients, their unaffected siblings and healthy controls: exploring putative endophenotypes. World J Biol Psychiatry. 2010;11:813-23.

12. Lopez C, Tchanturia K, Stahl D, Treasure J. Weak central coherence in eating disorders: a step towards looking for an endophenotype of eating disorders. J Clin Exp Neuropsychol. 2009;31:117-25.

13. Buhren K, Mainz V, Herpertz-Dahlmann B, Schafer K, Kahraman-Lanzerath B, Lente $\mathrm{C}$, et al. Cognitive flexibility in juvenile anorexia nervosa patients before and after weight recovery. J Neural Transm. 2012;119:1047-57.

14. Fitzpatrick KK, Darcy A, Colborn D, Gudorf C, Lock J. Set-shifting among adolescents with anorexia nervosa. Int J Eat Dis. 2012;45:909-12.

15. Shott ME, Filoteo JV, Bhatnagar KAC, Peak NJ, Hagman JO, Rockwell R, et al. Cognitive set-shifting in anorexia nervosa. Eur Eat Disord Rev. 2012;20:343-9.

16. Dahlgren $C L$, Lask $B$, Landrø NI, Rø Ø. Neuropsychological functioning in adolescents with anorexia nervosa before and after cognitive remediation therapy: A feasibility trial. Int J Eat Dis. 2013;46:576-81.

17. Lang K, Tchanturia K. A Systematic Review of Central Coherence in Young People with Anorexia Nervosa. J Child Adolesc Behav. 2014;2:140. doi:10.4172/jcalb.1000140.

18. Lang K, Stahl D, Espie J, Treasure J, Tchanturia K. Set shifting in children and adolescents with anorexia nervosa: An exploratory systematic review and meta-analysis. Int J Eat Dis. 2014;47:394-9.

19. Danner UN, Sanders N, Smeets PAM, Van Meer F, Adan RAH, Hoek HW, et al. Neuropsychological weaknesses in anorexia nervosa: Set-shifting, central coherence, and decision making in currently ill and recovered women. Int J Eat Dis. 2012;45:685-94.

20. Harrison A, Tchanturia K, Treasure J. Measuring state trait properties of detail processing and global integration ability in eating disorders. World J Biol Psychiatry. 2011:12:462-72.

21. Hatch A, Madden S, Kohn MR, Clarke S, Touyz S, Gordon E, et al. In first presentation adolescent anorexia nervosa, do cognitive markers of underweight status change with weight gain following a refeeding intervention? Int J Eat Dis. 2010;43:295-306.

22. Lezak MD, Howieson DB, Loring DW. Neuropsychological Assessment. 4th ed. Oxford: Oxford University Press; 2004. 
23. Sbordone RJ. Ecological validity: Some critical issues for the neuropsychologist. In: Sbordone RJ, Long CJ, editors. Ecological validity of neuropsychological testing. Delray Beach, FL, England: Gr Press/St Lucie Press; 1996. p. 15-41.

24. Amieva H, Phillips L, Della Sala S. Behavioral dysexecutive symptoms in normal aging. Brain Cog. 2003;53:129-32.

25. Bogod NM, Mateer CA, Macdonald SW. Self-awareness after traumatic brain injury: A comparison of measures and their relationship to executive functions. J Int Neuropsychol. 2003;9:450-8.

26. Norris G, Tate RL. The Behavioural Assessment of the Dysexecutive Syndrome (BADS): Ecological, concurrent and construct validity. Neuropsyc Rehabil. 2000;10:33-45.

27. Ready RE, Stierman L, Paulsen JS. Ecological validity of neuropsychological and personality measures of executive functions. Clin Neuropsychologist. 2001;15:314-23.

28. Vriezen ER, Pigott SE. The Relationship Between Parental Report on the BRIEF and Performance-Based Measures of Executive Function in Children with Moderate to Severe Traumatic Brain Injury. Child Neuropsychol. 2002:8:296-303.

29. Rose M, Davis J, Frampton I, Lask B. The Ravello Profile: development of a global standard neuropsychological assessment for young people with anorexia nervosa. Clin Child Psychol Psychiatry. 2011;16:195-202.

30. Rose M, Frampton I, Lask B. A case series investigating distinct neuropsychological profiles in children and adolescents with anorexia nervosa. Eur Eat Disord Rev. 2012;20:32-8.

31. Stedal K, Rose M, Frampton I, Landro NI, Lask B. The neuropsychological profile of children, adolescents, and young adults with anorexia nervosa. Arch Clin Neuropsych. 2012;27:329-37.

32. Van Noort BM, Pfeiffer E, Lehmkuhl U, Kappel V. An international neuropsychological assessment tool for children, adolescents, and adults with anorexia nervosa - The German adaptation of the Ravello Profile. Eine internationale neuropsychologische Testbatterie fur Kinder, Jugendliche und Erwachsene mit Anorexia nervosa - Die deutsche Adaptation des Ravello Profils. Z Kinder Jugendpsychiatr Psychother. 2013:41:371-81.

33. Chaytor N, Schmitter-Edgecombe M, Burr R. Improving the ecological validity of executive functioning assessment. Arch Clin Neuropsych. 2006:21:217-27.

34. Gioia GA, Isquith PK, Guy SC, Kenworthy L. Test Review. Behavior Rating Inventory of Executive Function. Child Neuropsycholh. 2000;6:235-8.

35. Gioia GA, Isquith PK. Ecological Assessment of Executive Function in Traumatic Brain Injury. Dev Neuropsychol. 2004;25:135-58.

36. Toplak ME, Bucciarelli SM, Jain U, Tannock R. Executive functions: performance-based measures and the behavior rating inventory of executive function (BRIEF) in adolescents with attention deficit/hyperactivity disorder (ADHD). Child Neuropsychol. 2008;15:53-72.

37. Mahone EM, Cirino PT, Cutting LE, Cerrone PM, Hagelthorn KM, Hiemenz JR, et al. Validity of the behavior rating inventory of executive function in children with ADHD and/or Tourette syndrome. Arch Clin Neuropsychol. 2002;17:643-62.

38. McCandless S, O' Laughlin L. The Clinical Utility of the Behavior Rating Inventory of Executive Function (BRIEF) in the diagnosis of ADHD. J Atten Disord. 2007;1:381-9.

39. Mangeot S, Armstrong K, Colvin AN, Yeates KO, Taylor HG. Long-term executive function deficits in children with traumatic brain injuries: Assessment using the Behavior Rating Inventory of Executive Function (BRIEF). Child Neuropsychol. 2002;8:271-84.

40. Mahone EM, Zabel TA, Levey E, Verda M, Kinsman S. Parent and self-report ratings of executive function in adolescents with myelomeningocele and hydrocephalus. Child Neuropsychol. 2002;8:258-70.

41. Dahlgren $C L$, Lask B, Landrø NI, Rø $\varnothing$. Patient and Parental Self-reports of Executive Functioning in a Sample of Young Female Adolescents with Anorexia Nervosa Before and After Cognitive Remediation Therapy. Eur Eat Disord Rev. 2014;22:45-52.

42. McAnarney ER, Zarcone J, Singh P, Michels J, Welsh S, Litteer T, et al. Restrictive Anorexia Nervosa and Set-Shifting in Adolescents: A Biobehavioral Interface. J Adolesc Health. 2011;49:99-101.

43. Guy SC. Behavior Rating Inventory of Executive Function-Self-Report version (BRIEF-SR). USA: Psychological Assessment Resources Inc.; 2004

44. Gioia GA. Behavior Rating Inventory of Executive Function: Professional manual. USA: Psychological Assessment Resources Inc.; 2000.
45. Fairburn CG. Eating disorder examination questionnaire (EDE-Q 6.0) Cognitive Behavior Therapy and Eating Disorders 2008.

46. Fairburn CG, Beglin SJ. Eating Disorder Examination Questionnaire (EDE-Q 6.0). In C.G. Fairburn (Ed.), Cognitive Behavior Therapy and Eating Disorders. New York: Guilford Press. 2008 p. 309-313.

47. Wechsler D. Wechsler Intelligence Scale for Children-Third Edition (WISC-III). San Antonio: The Psychological Corporation; 1992.

48. Wechsler D. Wechsler Abbreviated Scale of Intelligence (WASI) Manual. San Antonio: The Psychological Corporation; 1999.

49. Walker JM, D'Amato RC. Test Review: Behavior Rating Inventory of Executive Function-Self-Report Version. J Psychoeduc Assess. 2006;24:394-8.

50. Ciszewski S, Francis K, Mendella P, Bissada H, Tasca GA. Validity and reliability of the Behavior Rating Inventory of Executive Function - Adult Version in a clinical sample with eating disorders. Eat Behav. 2014;15:175-81.

51. Stedal K, Frampton I, Landro NI, Lask B. An examination of the ravello profile-a neuropsychological test battery for anorexia nervosa. Eur Eat Disord Rev. 2012;20:175-81.

52. Delis D, Kaplan E, Kramer J. Delis Kaplan Executiv Function System. San Antonio: The Psychological Corporation; 2001.

53. Meyers J, Meyers K. Rey Complex Figuer Test and Recognition Trial. Odessa: Psychological Assessment Resources Inc:; 1995.

54. Osterrieth PA. Le test de copie d'une figure complexe. Arch Psychol. 1944;30:206-356.

55. Burgess P, Shallice T. The Hayling and Brixton Test Manual. Bury St Edmunds: Thames Valley Test Company; 1997.

56. Chaytor N, Schmitter-Edgecombe M. The Ecological Validity of Neuropsychological Tests: A Review of the Literature on Everyday Cognitive Skills. Neuropsychol Rev. 2003;13:181-97.

57. Williams JM. A practical model of everyday assessment. In Sbordone, RJ, Charles $L$, editors. Ecological validity of neuropsychological testing. United States of America: CRC Press, 1996 p. 129-145.

58. Burgess PW, Alderman N, Evans J, Emslie H, Wilson BA. The ecological validity of tests of executive function. J Int Neuropsychol. 1998:4:547-58.

59. Mcauley T, Chen S, Goos L, Schachar R, Crosbie J. Is the behavior rating inventory of executive function more strongly associated with measures of impairment or executive function? J Int Neuropsychol. 2010;16:495-505.

60. Jeyakumar SLE, Warriner EM, Raval W, Ahmad SA. Balancing the Need for Reliability and Time Efficiency: Short Forms of the Wechsler Adult Intelligence Scale-III. Educ Psychol Meas. 2004;64:71-87.

61. Ryan JJ, Carruthers CA, Miller LJ, Souheaver GT, Gontkovsky ST, Zehr MD. Exploratory Factor Analysis of the Wechsler Abbreviated Scale of Intelligence (WASI) in Adult Standardization and Clinical Samples. App Neuropsychol. 2003;10:252-6.

62. Unterrainer JM, Rahm B, Kaller CP, Leonhart R, Quiske K, Hoppe-Seyler K, et al. Planning Abilities and the Tower of London: Is This Task Measuring a Discrete Cognitive Function? J Clin Ex Neuropsyc. 2004;26:846-56.

63. Kaller CP, Unterrainer JM, Rahm B, Halsband U. The impact of problem structure on planning: insights from the Tower of London task. Cog Brain Res. 2004:20:462-72.

64. Baughman FD, Cooper RP. Inhibition and young children's performance on the Tower of London task. Cog Sys Res. 2007:8:216-26.

65. Phillips LH. The Role of Memory in the Tower of London Task. Memory. 1999; : 209-31

66. Phillips L, Gilhooly K, Logie R, Della Sala S, Wynn V. Age, working memory, and the Tower of London task. Euro J Cog Psychol. 2003;15:291-312.

67. Chan RCK, Shum D, Toulopoulou T, Chen EYH. Assessment of executive functions: Review of instruments and identification of critical issues. Arch Clin Neuropsych. 2008;23:201-16.

68. Wilson BA. Ecological validity of neuropsychological assessment: Do neuropsychological indexes predict performance in everyday activities? App Prev Psychol. 1993;2:209-15.

69. Rabbitt P. Introduction: Methodologies and models in the study of executive function. In Rabbitt, P, editor. Methodology of frontal and executive function. Psychology Press, 2004, p.1-38.

70. Spooner DM, Pachana NA. Ecological validity in neuropsychological assessment: A case for greater consideration in research with neurologically intact populations. Arch Clin Neuropsych. 2006;21:327-37.

71. Vriezen E, Pigott S, Pelletier P. Developmental implications of early frontallobe damage: A case study. Brain Cog. 2001;47:222-5. 
72. Salimpoor VN, Desrocher M. Increasing the Utility of EF Assessment of Executive Function in Children. Dev Disabil. 2006;34:15-42.

73. Manchester D, Priestley N, Jackson H. The assessment of executive functions: Coming out of the office. Brain Inj. 2004;18:1067-81.

74. Shallice T, Burgess PW. Deficits in strategy application following frontal lobe damage in man. Brain. 1991;114:727-41.

75. Miotto EC, Bullock P, Polkey CE, Morris RG. Spatial working memory and strategy formation in patients with frontal lobe excisions. Cortex. 1996;32:613-30

76. Miotto EC, Morris RG. Virtual planning in patients with frontal lobe lesions. Cortex. 1998;34:639-57.

\section{Submit your next manuscript to BioMed Central} and take full advantage of:

- Convenient online submission

- Thorough peer review

- No space constraints or color figure charges

- Immediate publication on acceptance

- Inclusion in PubMed, CAS, Scopus and Google Scholar

- Research which is freely available for redistribution 